\title{
A SINGULAR ASYMPTOTIC EXPANSION FOR THE FIELD NEAR A MOVING DISLOCATION LOOP
}

\author{
BY \\ CONSTANTINE CALLIAS \\ (Case Western Reserve University, Cleveland, Ohio) \\ AND \\ XANTHIPPI MARKENSCOFF AND LUQUN NI ${ }^{1}$ \\ (University of California, San Diego, La Jolla, California)
}

\begin{abstract}
The coefficients of the $1 / \varepsilon$ and $\ln \varepsilon$ singular terms in the field quantities near an arbitrarily moving dislocation loop are obtained by singular asymptotic expansion of integrals.
\end{abstract}

Introduction. The stress fields radiated from a dislocation moving in an elastic solid have been studied since 1949 by Frank [7] and Eshelby [5] and subsequently by other investigators who studied steady state and accelerating motions of screw and edge dislocations in isotropic and anisotropic media. Some selected references include Eshelby [6], Kuisalaas and Mura [9, 10], Markenscoff and Clifton [13], and Markenscoff and Ni $[15,16]$. The motion of dislocation loops has also been studied. Mura [18] appears to be the first to formulate the problem in terms of the dynamic Green's function for an impulsive force in a three-dimensional space and to give the solution as an integral over the loop surface and over the loop history in time. $\mathrm{He}$ then converted the surface integral on the slip surface of the loop to a line integral along the loop curve. From a seismologist's point of view Burridge and Knopoff [2] formulated the problem in terms of surface integrals while Madariaga [11] treated an expanding circular fault numerically. Günther [8] and Mura [19] obtained solutions for uniformly moving loops while Markenscoff and Clifton [14], and Markenscoff and $\mathrm{Ni}[15,16]$ analyzed the wavefront of a circular dislocation loop starting from rest and expanding with constant radial velocity.

For general motion of a loop the solution is obtained only in terms of integrals over the history of the motion which cannot be evaluated in closed form; however, their asymptotic behavior may be appropriately analyzed. One limit which is of great importance is the near field, i.e., the field near the current position of the loop. In order to find this limiting behavior of the integrals, singular asymptotic series of

Received November 1, 1988.

${ }^{1}$ On leave from the Institute of Mathematics, Academia Sinica, Beijing, China. 
integrals are needed. Such an asymptotic series has been obtained previously (Callias and Markenscoff [3]) when the near field of an arbitrarily moving screw dislocation was obtained. The special feature of this solution is that it contains besides the $1 / \varepsilon$ singularity (with the same coefficient as in the steady-state motion), a $\ln \varepsilon$ singularity associated with the acceleration of the dislocation.

In this paper we formulate the solution to the generally moving loop (changing shape as well as position) in terms of a single integral around the loop curve, and present a method for reducing it to a form from which the singular asymptotic behavior is obtained by use of the asymptotic series of integrals. The coefficient of the $1 / \varepsilon$ and $\ln \varepsilon$ series are given explicitly. As an example the near field of an accelerating straight screw dislocation is obtained and compared to a known solution.

\section{Equations for a moving dislocation loop.}

A. Preliminaries. Mura [18] has given the equations giving the strains and velocity components for a moving loop as a double integral over the loop curve and time. These expressions, for the velocity and displacement gradients respectively, are

$$
\dot{U}_{m}(x, t)=\int_{-\infty}^{\infty} \int_{L\left(t^{\prime}\right)} C_{i j n l} G_{k m, l}\left(x-x^{\prime}, t-t^{\prime}\right) b_{i} \varepsilon_{j r h} V_{r}\left(x^{\prime}, f^{\prime}\right) d l_{h} d t^{\prime}
$$

and

$$
\begin{aligned}
U_{m, n}= & \int_{-\infty}^{\infty} \int_{L\left(t^{\prime}\right)} \varepsilon_{n j h} C_{i j k l} G_{k m, l}\left(x-x^{\prime}, t-t^{\prime}\right) b_{i} d l_{h} d t^{\prime} \\
& +\int_{-\infty}^{\infty} \int_{L\left(t^{\prime}\right)} \rho \dot{G}_{i m}\left(x-x^{\prime}, t-t^{\prime}\right) b_{i} \varepsilon_{n r s} V_{r}\left(x^{\prime}, t^{\prime}\right) d l_{s} d t^{\prime} .
\end{aligned}
$$

The equations include the case of a dislocation at rest which may have started moving at time $t^{\prime} \neq-\infty$; the initial static field is part of the solution. In Appendix $A$ it is shown that the solution for a screw dislocation being at rest and starting to move with constant velocity is retrieved from Eq. (2). Mura [9] did not use (2) directly but used (1) by integrating in time the velocity field and subsequently differentiating the displacement.

In expressions (1) and (2) $C_{i j k l}$ denote the elastic coefficients; $G_{k m}$, the Green's function for a unit impulse load in a full space; $V_{r}\left(\mathbf{x}, t^{\prime}\right)$, the velocity vector at any point of the loop; $b_{i}$ the Burgers vector; $d l_{h}$ a line element along the loop; and $\varepsilon_{j r s}$, the permutation symbol. For an isotropic material the Green's function is

$$
\begin{aligned}
G_{k m}\left(\mathbf{x}-\mathbf{x}^{\prime}, t-t^{\prime}\right)=\frac{1}{4 \pi \rho}\{ & \frac{\bar{t}}{\bar{r}^{2}}\left(\frac{3 \bar{r}_{k} \bar{r}_{m}}{\bar{r}^{3}}-\frac{\delta_{k m}}{\bar{r}}\right) H\left(\frac{\bar{r}}{c_{2}}-\bar{t}\right) H\left(\bar{t}-\frac{\bar{r}}{c_{1}}\right) \\
& \left.+\frac{\bar{r}_{k} \bar{r}_{m}}{\bar{r}^{3}}\left[\frac{1}{c_{1}^{2}} \delta\left(\bar{t}-\frac{\bar{r}}{c_{1}}\right)-\frac{1}{c_{2}^{2}} \delta\left(\bar{t}-\frac{\bar{r}}{c_{2}}\right)\right]+\frac{\delta_{k m}}{c_{2}^{2} \bar{r}} \delta\left(\bar{t} \frac{\bar{r}}{c_{2}}\right)\right\},
\end{aligned}
$$

where $\rho$ is the density, $\bar{t}=t-t^{\prime}, \bar{r}_{i}=x_{i}-x_{i}^{\prime}, r^{2}=x_{1}^{2}+x_{2}^{2}+x_{3}^{2}, \bar{r}^{2}=\left(x_{1}-x_{1}^{\prime}\right)^{2}+$ $\left(x_{2}-x_{2}^{\prime}\right)^{2}+\left(x_{3}-x_{3}^{\prime}\right)^{2} ; c_{1}$ and $c_{2}$ are the longitudinal and shear wave speeds, $H(\cdot)$ denotes the Heaviside step function, $\delta(\cdot)$ denotes the delta function.

B. Integrations of the line and time integrals. Integration in the expressions (1) and (2) may be performed in time first and space second. This has the advantage that for subsonic motion the denominator never vanishes and thus convergence of 
the integral is always guaranteed, except possibly for infinite loops, in which case for straight dislocations, it can easily be seen that the integral converges as well. However, as it becomes obvious in the sequel, $\partial \mathbf{x}^{\prime}\left(t^{\prime}\right) / \partial t^{\prime}$ must be piecewise smooth. In the case of a loop being at rest and starting to move at $t^{\prime}=0$ with finite velocity $V$, this derivative is discontinuous at $t^{\prime}=0$ and the integral has to be split into two parts, i.e., $\int_{-\infty}^{0} d t^{\prime}+\int_{0}^{t} d t^{\prime}$.

Starting from an original time $t_{0} \geq-\infty$ the loop $L\left(t_{0}\right)$ can be parametrized by a parameter $\sigma: \alpha \leq \sigma \leq \beta$. At time $t^{\prime}$ the point $\mathbf{x}^{\prime}\left(\sigma, t_{0}\right)$ is at the position $\mathbf{x}^{\prime}\left(\sigma, t^{\prime}\right)$ which is determined, if the velocity of the loop is known, according to

$$
\mathbf{x}^{\prime}\left(\sigma, t^{\prime}\right)=\mathbf{x}\left(\sigma, t_{0}\right)+\int_{t_{0}}^{t^{\prime}} \mathbf{V}(\sigma, \tau) d \tau
$$

We define a curve $\Gamma_{i}$ on the surface described by the moving loop (see Fig. 2) that satisfies the equation

$$
\left|\mathbf{x}-\mathbf{x}^{\prime}\left(\sigma, t^{\prime}\right)\right|=c_{i}\left(t-t^{\prime}\right), \quad i=1,2
$$

(with $c_{1}$ being the longitudinal wave speed and $c_{2}$ the shear one).

For a given $\sigma$, Eq. (4) defines a unique $t^{\prime}=\tau(\sigma)$, i.e.,

$$
c(t-\tau(\sigma))=\bar{r}=\left|\mathbf{x}-\mathbf{x}^{\prime}(\sigma, \tau(\sigma))\right| .
$$

Since $\tau(\sigma)$ is a function of $(\mathbf{x}, t)$, we have

$$
\frac{\partial \tau}{\partial x_{l}}=\frac{-\bar{r}_{l}}{c \bar{r}-\left(\overline{\mathbf{r}}^{\prime}, \partial \mathbf{x}^{\prime} / \partial t^{\prime}\right)} \equiv \frac{-\bar{r}_{l}}{\Delta_{1}}
$$

where $\Delta_{1} \equiv c \bar{r}-\left(\overline{\mathbf{r}},\left(\partial / \partial t^{\prime}\right) \mathbf{x}^{\prime}\right)$ and $\left(\overline{\mathbf{r}},(\partial / \partial t) \mathbf{x}^{\prime}\right)$ denotes the inner product of the vectors $\mathbf{r}$ and $\partial \mathbf{x}^{\prime} / \partial t^{\prime}$, and

$$
\frac{\partial \tau}{\partial t}=\frac{c \bar{r}}{\Delta_{1}} .
$$

For further use we note that for $\bar{r}=\bar{r}(\mathbf{x}, \sigma, \tau(\sigma))$

$$
\begin{gathered}
\frac{\partial \bar{r}}{\partial x_{l}}=\frac{\bar{r}_{l}+\left(\overline{\mathbf{r}}, \partial x^{\prime} / \partial t^{\prime}\right) \bar{r}_{l} / \Delta_{1}}{\bar{r}}=\frac{c \bar{r}_{l}}{\Delta_{l}} \\
\frac{\partial \Delta_{1}}{\partial x_{l}}=\frac{\partial}{\partial x_{l}}\left[c \bar{r}-\left(\overline{\mathbf{r}}, \frac{\partial \mathbf{x}^{\prime}}{\partial t^{\prime}}\right)\right]=\frac{\bar{r}_{l}}{\Delta_{1}}\left[c^{2}-\left(\frac{\partial \mathbf{x}^{\prime}}{\partial t^{\prime}}, \frac{\partial \mathbf{x}^{\prime}}{\partial t^{\prime}}\right)+\left(\overline{\mathbf{r}}, \frac{\partial^{2} \mathbf{x}^{\prime}}{\partial t^{\prime 2}}\right)\right]-\left(\frac{\partial x_{l}}{\partial t^{\prime}}\right) .
\end{gathered}
$$

In performing the integrations indicated in (1) and (2) let us consider first the terms of the Green's function

$$
\frac{\bar{t}}{4 \pi \rho \bar{r}^{2}}\left(3 \bar{r}_{k} \frac{\bar{r}_{m}}{\bar{r}^{3}}-\frac{\partial_{k m}}{\bar{r}}\right) H\left(\frac{\bar{r}}{c}-\bar{t}\right)-\frac{\bar{r}_{k} \bar{r}_{m}}{4 \pi \rho \bar{r}^{3} c^{2}} \delta\left(\bar{t}-\frac{\bar{r}}{c}\right) \equiv \hat{G}_{k m}(c)
$$

and interchange the orders of integrations in time and space and write

$$
\begin{aligned}
\mathrm{I} & \equiv \int_{-\infty}^{t} d t \int_{L\left(t^{\prime}\right)} \varepsilon_{n j h} C_{i j k l} \hat{G}_{k m, l} b_{i} d l_{h} \\
& =\varepsilon_{n j h} C_{i j k l} b_{i} \int_{\alpha}^{\beta} d \sigma \int_{-\infty}^{t} \hat{G}_{k m}\left(\frac{\partial}{\partial \sigma} \mathbf{x}^{\prime}\right)_{h} d t^{\prime} .
\end{aligned}
$$


For the term

$$
\mathrm{II} \equiv \int_{-\infty}^{t} d t^{\prime} \int_{L\left(t^{\prime}\right)} b_{i} \rho \varepsilon_{n r s} \hat{G}_{i m} V_{r}\left(t^{\prime}\right) d l_{s}
$$

we can perform the differentiation inside the integral according to Leibnitz's rule and obtain

$$
\begin{array}{r}
\mathrm{II}=\frac{b_{i} \rho \varepsilon_{n r s}}{4 \pi \rho} \int_{\alpha}^{\beta} \frac{\partial}{\partial t}\left[\int_{\tau}^{t} \frac{\bar{t}}{\bar{r}^{2}}\left(\frac{3 \bar{r}_{i} \bar{r}_{m}}{\bar{r}^{3}}-\frac{\delta_{i m}}{\bar{r}}\right)\left(\frac{\partial}{\partial t^{\prime}} \mathbf{x}^{\prime}\right)_{r}\left(\frac{\partial}{\partial \sigma} \mathbf{x}^{\prime}\right)_{s} d t^{\prime}\right. \\
\left.-\left.\frac{\bar{r}_{i} \bar{r}_{m}}{\bar{r}^{2} c} \frac{\left(\partial / \partial t^{\prime}\right)\left(\mathbf{x}^{\prime}\right)_{r}\left((\partial / \partial \sigma) \mathbf{x}^{\prime}\right)_{s}}{\Delta_{1}}\right|_{t^{\prime}=\tau}\right] d \sigma .
\end{array}
$$

Thus the terms I and II of the strain combined give

$$
\begin{aligned}
& \int_{\alpha}^{\beta} d \sigma\left[\left\{\frac{(\partial / \partial \sigma) x_{h}^{\prime}}{c \bar{r}^{2} \Delta_{l}^{2}}\left(\bar{r}_{l} \bar{r}_{m} \frac{\partial x_{k}^{\prime}}{\partial t^{\prime}}+\bar{r}_{l} \bar{r}_{k} \frac{\partial x_{m}^{\prime}}{\partial l^{\prime}}+\bar{r}_{k} \bar{r}_{m} \frac{\partial x_{l}^{\prime}}{\partial t^{\prime}}\right)\right.\right. \\
& +\frac{(\partial / \partial \sigma) x_{h}^{\prime}}{c \bar{r}^{2} \Delta_{l}}\left(\delta_{k m} \bar{r}_{l}+\delta_{m l} \bar{r}_{k}+\delta_{k l} \bar{r}_{m}\right)-\frac{2(\partial / \partial \sigma) x_{h}^{\prime}}{\bar{r}^{2} \Delta_{1}^{2}} \bar{r}_{k} \bar{r}_{m} \bar{r}_{l} \\
& -\frac{(\partial / \partial \sigma) x_{h}^{\prime}}{c \bar{r}^{2} \Delta_{1}^{3}} \bar{r}_{k} \bar{r}_{m} \bar{r}_{l}\left(c^{2}-\left(\frac{\partial \mathbf{x}^{\prime}}{\partial t^{\prime}}, \frac{\partial \mathbf{x}^{\prime}}{\partial t^{\prime}}\right)+\left(\mathbf{r}, \frac{\partial^{2} \mathbf{x}^{\prime}}{\partial t^{\prime 2}}\right)\right) \\
& \left.-\frac{3(\partial / \partial \sigma) x_{h}^{\prime}}{c \bar{r}^{4} \Delta_{1}} \bar{r}_{k} \bar{r}_{m} \bar{r}_{l}-\frac{\left(\partial^{2} / \partial \sigma \partial t^{\prime}\right) x_{h}^{\prime}}{c \bar{r}^{2} \Delta_{l}^{2}} \bar{r}_{k} \bar{r}_{m} \bar{r}_{l}\right\}\left(-\frac{\varepsilon_{n j k} C_{i j k l} b_{i}}{4 \pi \rho}\right) \\
& +\left\{\frac{\bar{r}_{i} \bar{r}_{m}}{\bar{r} \Delta_{1}^{2}}\left(\left(\frac{\partial^{2}}{\partial t^{\prime 2}} \mathbf{x}^{\prime}\right)_{r}\left(\frac{\partial}{\partial \sigma} \mathbf{x}^{\prime}\right)_{s}+\left(\frac{\partial}{\partial t^{\prime}} \mathbf{x}^{\prime}\right)_{r}\left(\frac{\partial^{2}}{\partial \sigma \partial t^{\prime}} \mathbf{x}^{\prime}\right)_{s}\right)\right. \\
& +\left(\frac{\partial}{\partial t^{\prime}} \mathbf{x}^{\prime}\right)_{r}\left(\frac{\partial}{\partial \sigma} \mathbf{x}^{\prime}\right)_{s}\left[\frac{(-)\left(\left(\partial x_{i}^{\prime} / \partial t^{\prime}\right) \bar{r}_{m}+\left(\partial x_{m}^{\prime} / \partial t^{\prime}\right) \bar{r}_{i}\right)}{\bar{r}_{1}^{2}}+\frac{3 c \bar{r}_{i} \bar{r}_{m}}{\bar{r}^{2} \Delta_{1}^{2}}\right. \\
& -\frac{\delta_{i m}}{\bar{r} \Delta_{1}}+\frac{\bar{r}_{i} \bar{r}_{m}\left(\left(\bar{r}, \partial \mathbf{x}^{\prime}\right) / \partial t^{\prime}\right)^{2}}{\bar{r}^{3} \Delta_{1}^{3}}-\frac{\bar{r}_{i} \bar{r}_{m}}{\bar{r} \Delta_{1}^{3}}\left(\frac{\partial \mathbf{x}^{\prime}}{\partial t^{\prime}}, \frac{\partial \mathbf{x}^{\prime}}{\partial t^{\prime}}\right) \\
& \left.\left.\left.+\frac{\bar{r}_{i} \bar{r}_{m}}{\bar{r} \Delta_{1}^{3}}\left(\mathbf{r}, \frac{\partial^{2} \mathbf{x}^{\prime}}{\partial t^{\prime 2}}\right)\right]\right\}\left(-\frac{b_{i} \varepsilon_{n r s}}{4 \pi}\right)\right] \\
& +\int_{\alpha}^{\beta} d \sigma \int_{\tau}^{t} d t^{\prime}\left[\bar{t}\left(\frac{\partial}{\partial \sigma} \mathbf{x}^{\prime}\right)_{h}\left[\frac{3\left(\delta_{k m} \bar{r}_{l}+\delta_{k l} \bar{r}_{m}+\delta_{m l} \bar{r}_{k}\right)}{\bar{r}^{5}}-\frac{15 \bar{r}_{k} \bar{r}_{m} \bar{r}_{l}}{\bar{r}^{7}}\right]\right. \\
& \left.\cdot\left(\frac{\varepsilon_{n j h} C_{i j k l} b_{i}}{4 \pi \rho}\right)+\left[\left(\frac{\partial}{\partial t^{\prime}} \mathbf{x}^{\prime}\right)_{r}\left(\frac{\partial}{\partial \sigma} \mathbf{x}^{\prime}\right)_{s}\left(3 \frac{\bar{r}_{i} \bar{r}_{m}}{\bar{r}^{5}}-\frac{\delta_{i m}}{\bar{r}^{3}}\right)\right]\left(\frac{b_{i} \varepsilon_{n r s}}{4 \pi}\right)\right] .
\end{aligned}
$$

Since from (10),

$$
G_{k m}=\hat{G}_{k m}\left(c_{2}\right)-\hat{G}_{k m}\left(c_{1}\right)+\frac{\delta_{k m} \delta\left(\bar{t}-\left(\bar{r} / c_{2}\right)\right)}{4 \pi \rho c_{2}^{2} \bar{r}}
$$


we have the additional contribution to the strain from the last term of (13)

$$
\begin{gathered}
\frac{\partial}{\partial x_{l}} \int_{-\infty}^{t} d t^{\prime} \int_{L\left(t^{\prime}\right)} \varepsilon_{n j h} C_{i j k l}\left(\frac{\delta_{k m}}{4 \pi \rho c_{2}^{2} \bar{r}} \delta\left(\bar{t}-\frac{\bar{r}}{c_{2}}\right)\right) b_{i} d l_{h} \\
+\frac{\partial}{\partial t} \int_{-\infty}^{t} d t^{\prime} \int_{L\left(t^{\prime}\right)} b_{i} \rho\left(\frac{\delta_{i m}}{4 \pi \rho c_{2}^{2} \bar{r}} \delta\left(\bar{t}-\frac{\bar{r}}{c_{2}}\right)\right) \varepsilon_{n r s} V_{r} d l_{s} \\
=\int_{\alpha}^{\beta} d \sigma\left[( \frac { \varepsilon _ { n j h } C _ { i j k l } b _ { i } } { 4 \pi \rho c _ { 2 } } ) \left[\frac{-\bar{r}_{l}\left(\left(\partial^{2} / \partial \sigma \partial t^{\prime}\right) x_{h}^{\prime}\right)}{\Delta_{1}^{2}}+\frac{\left((\partial / \partial \sigma) x_{h}^{\prime}\right)\left(\left(\partial / \partial t^{\prime}\right) x_{1}^{\prime}\right)}{\Delta_{1}^{2}}\right.\right. \\
+\left(\frac{b_{i} \varepsilon_{n r s}}{4 \pi c_{2}}\right)\left[\frac{c \bar{r}}{\Delta_{1}^{2}}\left(\left(\frac{\partial^{2}}{\partial \sigma \partial t^{\prime}} x_{h}^{\prime}\right)\left(\frac{\partial}{\partial t^{\prime}} x_{r}^{\prime}\right)+\left(\frac{\partial}{\partial \sigma} x_{h}^{\prime}\right)\left(\frac{\partial^{2}}{\partial \sigma \partial t^{\prime}} x_{r}^{\prime}\right)\right)\right. \\
+\frac{c \bar{r}}{\Delta_{1}^{3}}\left(\frac{c\left(\mathbf{r},\left(\partial \mathbf{x}^{\prime} / \partial t^{\prime}\right)\right)}{\bar{r}}-\left(\frac{\partial \mathbf{x}^{\prime}}{\partial t^{\prime}}, \frac{\partial \mathbf{x}^{\prime}}{\partial t^{\prime}}\right)+\left(\mathbf{r}, \frac{\partial^{2} \mathbf{x}^{\prime}}{\partial t^{\prime 2}}\right)\right) \\
\left.\left.\times\left(\frac{\partial \mathbf{x}^{\prime}}{\partial t^{\prime}}, \frac{\partial \mathbf{x}^{\prime}}{\partial t^{\prime}}\right)+\left(\mathbf{r}, \frac{\partial^{2} \mathbf{x}^{\prime}}{\partial t^{\prime 2}}\right)\right)\right]
\end{gathered}
$$

\section{Evaluation of the stress near the loop.}

A. Preliminaries. Let us assume that $L\left(t^{\prime}\right)$ is a smooth loop which describes, as it moves, the surface $\mathbf{x}^{\prime}\left(t^{\prime}\right)$. A point $\Sigma$ is a fixed physical point with coordinate $\mathbf{x}_{0}(t)$ on $L(t)$ moving with the loop. A field point $P$ with coordinates $\mathbf{x}(t)$ is near the moving loop. The distance between $P$ and $\Sigma$ is of $O(\varepsilon)$, i.e., $\mathbf{x}(t)-\mathbf{x}_{0}^{\prime}(t)=\varepsilon \zeta$, where $\zeta$ is a fixed vector. What we want to achieve in this paper is to obtain the singular part of the stress at the field point $P$ as $\varepsilon \rightarrow 0$, i.e., as the point $P$ approaches the loop (at the point $\Sigma$ ). Since the solution as given in Eq. (16) is in the form of an integral with the integrand being a function of $\varepsilon$, we need to obtain the asymptotic expansions of an integral in $\varepsilon$. However Taylor series expansions in powers of $\varepsilon$ of the integrand would give divergent coefficients of the powers of $\varepsilon$, and thus regular asymptotics do not work. We observe that we can bring the integral to a form

$$
\int_{\sigma_{0}} F\left(\sigma-\sigma_{0}, \frac{\varepsilon}{\sigma-\sigma_{0}}\right) d \sigma
$$

with $\sigma_{0}$ being the singular point (or the point $\Sigma$ in Fig. 1), and the asymptotic series given in Callias and Markenscoff [3] can be directly applied. Since this is the main tool of our technique we state it again here.

TheOREM 1. Under certain restrictions in Callias and Markenscoff [3] for the function $h(x, y)$, where $y=\varepsilon / x$, and for $0<b \leq \infty$ we have

$$
\int_{0}^{b} h(x, y) d x \sim A_{0}+\sum_{m=1}^{\infty} A_{m} \varepsilon^{m}+\ln \varepsilon \sum_{m=1}^{\infty} B_{m} \varepsilon^{m}, \quad \text { as } \varepsilon \rightarrow 0
$$


where

$$
\begin{gathered}
A_{0}=\int_{0}^{b} d x h(x, 0), \\
A_{m}=u_{m}(h)+L_{m}(h)+\frac{1}{m !(m-1) !} \partial_{y}^{m-1} \partial_{y}^{m} h(0,0) \sum_{j=1}^{m-1} \frac{1}{j} \\
+\sum_{j=1}^{m}(-1)^{m+j} \frac{(j-1) !}{m !(m-1) !} \frac{1}{b^{j}} \partial_{x}^{m-1-j} \partial_{y}^{m} h(b, 0), \\
B_{m}=\frac{1}{m !(m-1) !} \partial_{x}^{m-1} \partial_{y}^{m} h(0,0),
\end{gathered}
$$

and

$$
\begin{aligned}
u_{m}(h) & =-\frac{1}{m !(m-1) !} \int_{0}^{\infty} \ln x \partial_{x}^{m} \partial_{y}^{m} h(x, 0) d x \\
L_{m}(h) & =-\frac{1}{(m-1) !} \int_{0}^{\infty} \ln \xi \partial_{\xi}\left[\xi^{m} \partial_{x}^{m-1} R_{m+1}\left(0, \frac{1}{\xi}\right)\right] d \xi \\
& =\lim _{\delta \rightarrow 0}\left\{\int_{\delta}^{\infty} \xi^{m-1} \partial_{x}^{m-1} R_{m+1}\left(0, \frac{1}{\xi}\right) d \xi-\ln \xi \cdot \frac{1}{m !} \partial_{x}^{m-1} \partial_{y}^{m} h(0,0)\right\}
\end{aligned}
$$

with $R_{m+1}(x, y)$ as the remainder in the Taylor series of $h(x, y)$ about $y=0$

$$
R_{m+1}(x, y)=h(x, y)-\sum_{k=0}^{m} \frac{1}{k !} \partial_{y}^{k} h(x, 0) y^{k} .
$$

B. Expansion of the integrand about the singular point. We are now going to bring the integral to the form

$$
\int_{\sigma_{0}} F\left(\sigma-\sigma_{0}, \frac{\varepsilon}{\sigma-\sigma_{0}}\right) d \sigma
$$

and for that we need to expand every term in (16) and (18) about the point $\left(\sigma_{0}, t\right)$. This procedure is described in the sequel.

A point $M\left(\mathbf{x}^{\prime}(\sigma, \tau(\sigma))\right)$ on the curve $\Gamma$ will be at the position $N\left(\mathbf{x}^{\prime}(\sigma, t)\right)$ at time $t$ (see Fig. 1), so that we can write $\mathbf{M P}=\mathbf{\Sigma} \mathbf{P}+\mathbf{N} \boldsymbol{\Sigma}+\mathbf{M N}$, or

$$
\mathbf{r}=\mathbf{x}-\mathbf{x}^{\prime}(\sigma, \tau(\sigma))=\mathbf{x}-\mathbf{x}^{\prime}\left(\sigma_{0}, t\right)+\mathbf{x}^{\prime}\left(\sigma_{0}, t\right)-\mathbf{x}(\sigma, t)+\mathbf{x}^{\prime}(\sigma, t)-\mathbf{x}^{\prime}(\sigma, \tau(\sigma)) .
$$

Moreover we can expand in powers of $\sigma-\sigma_{0}$ and $t-\tau(\sigma)$ to write

$$
\begin{gathered}
\mathbf{x}-\mathbf{x}^{\prime}\left(\sigma_{0}, t\right)=\mathbf{x}-\mathbf{x}_{0}(t) \equiv \varepsilon \zeta, \\
\mathbf{x}^{\prime}\left(\sigma_{0}, t\right)-\mathbf{x}^{\prime}(\sigma, t)=\omega\left(\sigma-\sigma_{0}\right),
\end{gathered}
$$

where

$$
\begin{gathered}
\boldsymbol{\omega} \equiv-\left\{\left.\frac{\partial}{\partial \sigma} \mathbf{x}^{\prime}(\sigma, t)\right|_{\sigma=\sigma_{0}}+\left.\frac{1}{2} \frac{\partial^{2}}{\partial \sigma^{2}}\left(\mathbf{x}^{\prime}(\sigma, t)\right)\right|_{\sigma=\sigma_{0}}(\sigma-\sigma)+\cdots\right\}, \\
\mathbf{x}^{\prime}(\sigma, t)-\mathbf{x}^{\prime}(\sigma, \tau(\sigma))=\mathbf{m}(t-\tau(\sigma)),
\end{gathered}
$$

with

$$
\mathbf{m} \equiv \frac{\partial}{\partial t} \mathbf{x}^{\prime}(\sigma, t)-\frac{1}{2} \frac{\partial^{2}}{\partial t^{2}} \mathbf{x}^{\prime}(\sigma, t)(t-\tau(\sigma))+\cdots
$$




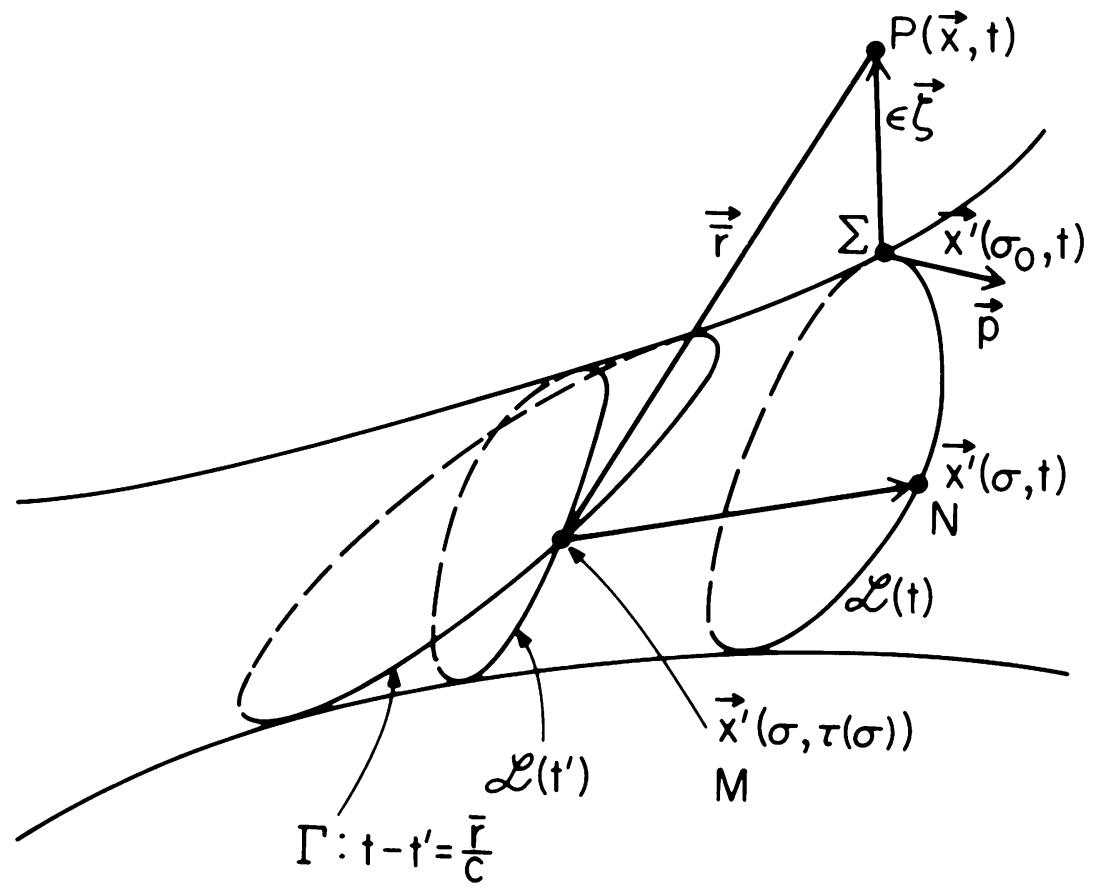

Fig. 1.

According to the relation of Eq. (4), $\tau$ is a function of $\sigma$; however, we cannot simply expand $(t-\tau)$ in powers of $\left(\sigma-\sigma_{0}\right)$, as it would seem the natural thing to do at this point, since the derivative $d^{2} \tau / d \sigma^{2}$ may not exist as $\varepsilon \rightarrow 0$, at $\sigma=\sigma_{0}$. So there is no reason to believe this expansion is convergent. We show this at the example of a circular loop moving with velocity $v$ and at a field point at a distance $\varepsilon$ above the loop, i.e., for $x_{1}=V t H(t), x_{2}=0, x_{3}=\varepsilon, x_{1}^{\prime}=V t^{\prime} H\left(t^{\prime}\right) \cos \theta, x_{2}^{\prime}=V t^{\prime} H\left(t^{\prime}\right) \sin \theta, x_{3}^{\prime}=0$. The equation for $\Gamma: \mathbf{x}-\mathbf{x}^{\prime}=c\left(t-t^{\prime}\right)$ gives $V^{2}\left(t-t^{\prime} \cos \theta\right)^{2}+V^{2} t^{\prime 2} \sin ^{2} \theta+\varepsilon^{2}=c^{2}\left(t-t^{\prime}\right)^{2}$ with root

$$
t^{\prime}=\frac{\left(c^{2}-V^{2} \cos \theta\right) t \pm \sqrt{\left[\left(c^{2}-V^{2} \cos \theta\right)^{2}-\left(c^{2}-V^{2}\right)^{2}\right] t^{2}+\varepsilon^{2}\left(c^{2}-V^{2}\right)}}{c^{2}-V^{2}} .
$$

Then at $\theta=0$

$$
\frac{\partial^{2} t^{\prime}}{\partial \theta^{2}}= \pm \frac{t^{2} V^{2}}{\sqrt{\left(c^{2}-V^{2}\right) \varepsilon}}+\frac{V^{2}}{c^{2}-V^{2}}
$$

diverges as $\varepsilon \rightarrow 0$.

We then define the new variable

$$
S \equiv \frac{t-\tau(\sigma)}{\left|\varepsilon \zeta+\left(\sigma-\sigma_{0}\right) \boldsymbol{\omega}\right|}=\frac{t-\tau(\sigma)}{\left|\sigma-\sigma_{0}\right||\zeta+\omega|}
$$

and it is easy to see, as we will calculate later, that the derivatives

$$
\frac{\partial^{i+j} S}{\partial \sigma^{i} \partial y^{i}} \sim O(1) \quad \text { as } y \rightarrow 0 \text { and } \sigma \rightarrow \sigma_{0}, i, j=1,2,3, \ldots
$$


We express the terms in the integrand as functions of the variables, $\theta \equiv \sigma-\sigma_{0}, y, S$. We have from (15), (16), (17), (18)

$$
\begin{aligned}
\overline{\mathbf{r}} & =\varepsilon \boldsymbol{\zeta}+\left(\sigma-\sigma_{0}\right) \boldsymbol{\omega}+\mathbf{m} S\left|\varepsilon \boldsymbol{\beta}+\left(\sigma-\sigma_{0}\right) \boldsymbol{\omega}\right| \\
& =\left(\sigma-\sigma_{0}\right)(y \boldsymbol{\beta}+\boldsymbol{\omega})+\mathbf{m}_{1}\left|\sigma-\sigma_{0}\right||y \boldsymbol{\beta}+\boldsymbol{\omega}|
\end{aligned}
$$

with

$$
\begin{aligned}
& \mathbf{m}_{1} \equiv \mathbf{m} S=\frac{\partial}{\partial t} \mathbf{x}^{\prime}\left(\sigma_{0}, t\right) S+\frac{\partial^{2}}{\partial \sigma \partial t} \mathbf{x}^{\prime}\left(\sigma_{0}, t\right) S\left(\sigma-\sigma_{0}\right) \\
& -\frac{1}{2} \frac{\partial^{2}}{\partial t^{2}} \mathbf{x}^{\prime}\left(\sigma_{0}, t\right) S^{2}|y \zeta+\omega|\left|\sigma-\sigma_{0}\right|+\text { h.o.t. of }\left(\sigma-\sigma_{0}\right), \\
& \frac{\partial}{\partial \sigma} \mathbf{x}^{\prime}(\sigma, \tau(\sigma))=\frac{\partial}{\partial \sigma} \mathbf{x}^{\prime}\left(\sigma_{0}, t\right)+\frac{\partial^{2}}{\partial \sigma^{2}} \mathbf{x}^{\prime}\left(\sigma_{0}, t\right)\left(\sigma-\sigma_{0}\right) \\
& -\frac{\partial^{2}}{\partial \sigma \partial t} \mathbf{x}^{\prime}\left(\sigma_{0}, t\right) S|y \boldsymbol{\zeta}+\boldsymbol{\omega}|\left|\sigma-\sigma_{0}\right|+\text { h.o.t., } \\
& \left.\frac{\partial}{\partial t^{\prime}} \mathbf{x}^{\prime}\left(\sigma, t^{\prime}\right)\right|_{t^{\prime}=\tau(\sigma)}=\frac{\partial}{\partial t} \mathbf{x}\left(\sigma_{0}, t\right)+\frac{\partial^{2}}{\partial \sigma \partial t} \mathbf{x}^{\prime}\left(\sigma_{0}, t\right)\left(\sigma-\sigma_{0}\right)-\frac{\partial^{2}}{\partial t^{2}} \mathbf{x}^{\prime}\left(\sigma_{0}, t\right) S|y \zeta+\omega|\left|\sigma-\sigma_{0}\right|, \\
& \frac{\partial^{2}}{\partial \sigma \partial t} \mathbf{x}^{\prime}(\sigma, \tau(\sigma))=\frac{\partial^{2}}{\partial \sigma \partial t} \mathbf{x}^{\prime}\left(\sigma_{0}, t\right)+\frac{\partial^{3}}{\partial \sigma^{2} \partial t} \mathbf{x}^{\prime}\left(\sigma_{0}, t\right)\left(\sigma-\sigma_{0}\right) \\
& -\frac{\partial^{3}}{\partial \sigma \partial t^{2}} \mathbf{x}^{\prime}\left(\sigma_{0}, t\right) S\left|\sigma-\sigma_{0}\right||y \zeta+\omega|+\text { h.o.t., } \\
& \left.\frac{\partial^{2}}{\partial t^{\prime 2}} \mathbf{x}^{\prime}\left(\sigma, t^{\prime}\right)\right|_{t^{\prime}=\tau(\sigma)}=\frac{\partial^{2}}{\partial t^{2}} \mathbf{x}^{\prime}\left(\sigma_{0}, t\right)+\frac{\partial^{3}}{\partial t^{2} \partial \sigma} \mathbf{x}^{\prime}\left(\sigma_{0}, t\right)(\sigma-\sigma) \\
& -\frac{\partial^{3}}{\partial t^{3}} \mathbf{x}^{\prime}\left(\sigma_{0}, t\right) S\left|\sigma-\sigma_{0}\right||y \zeta+\boldsymbol{\omega}|+\text { h.o.t., } \\
& r=c S\left|\sigma-\sigma_{0}\right||y \zeta+\omega|, \\
& \Delta_{1}=c r-\left(\mathbf{r}, \frac{\partial}{\partial t^{\prime}} \mathbf{x}^{\prime}\right)=\left[c^{2}-\left(\frac{\partial}{\partial t} \mathbf{x}^{\prime}\left(\sigma_{0}, t\right)\right)^{2}\right] S\left|\sigma-\sigma_{0}\right||y \boldsymbol{\zeta}+\boldsymbol{\omega}| \\
& -\left(y \boldsymbol{\zeta}+\boldsymbol{\omega}, \frac{\partial}{\partial t} \mathbf{x}^{\prime}\left(\sigma_{0}, t\right)\right)\left(\sigma-\sigma_{0}\right) \\
& -2\left(\frac{\partial^{2}}{\partial \sigma \partial t} \mathbf{x}^{\prime}\left(\sigma_{0}, t\right), \frac{\partial}{\partial t} \mathbf{x}^{\prime}\left(\sigma_{0}, t\right)\right) S\left(\sigma-\sigma_{0}\right)\left|\sigma-\sigma_{0}\right||y \boldsymbol{\zeta}+\boldsymbol{\omega}| \\
& +\frac{3}{2}\left(\frac{\partial^{2}}{\partial t^{2}} \mathbf{x}^{\prime}\left(\sigma_{0}, t\right), \frac{\partial}{\partial t} \mathbf{x}^{\prime}\left(\sigma_{0}, t\right)\right) S^{2}\left|\sigma-\sigma_{0}\right|^{2}|y \zeta+\omega|^{2} \\
& -\left(y \boldsymbol{\zeta}+\boldsymbol{\omega}, \frac{\partial^{2}}{\partial \sigma \partial t} \mathbf{x}^{\prime}\left(\sigma_{0}, t\right)\right)\left(\sigma-\sigma_{0}\right)^{2} \\
& +\left(\frac{\partial^{2}}{\partial t^{2}} \mathbf{x}^{\prime}\left(\sigma_{0}, t\right), y \zeta+\omega\right) S\left(\sigma-\sigma_{0}\right)\left|\sigma-\sigma_{0}\right||y \zeta+\omega|+\text { h.o.t. }
\end{aligned}
$$

In order to simplify the notation of the final expressions we introduce the following symbols needed to describe the surface described by $L(t)$ at the point $\left(\sigma_{0}, t\right)$

$$
\frac{\partial}{\partial t} \mathbf{x}^{\prime}\left(\sigma_{0}, t\right)=\mathbf{v}
$$




$$
\begin{gathered}
\frac{\partial^{2}}{\partial t^{2}} \mathbf{x}^{\prime}\left(\sigma_{0}, t\right)=\mathbf{a}, \\
\frac{\partial}{\partial \sigma} \mathbf{x}^{\prime}\left(\sigma_{0}, t\right)=\mathbf{p}, \\
\frac{\partial^{2}}{\partial \sigma^{2}} \mathbf{x}^{\prime}\left(\sigma_{0}, t\right)=\mathbf{q}, \\
\frac{\partial^{2}}{\partial \sigma \partial t} \mathbf{x}^{\prime}\left(\sigma_{0}, t\right)=\mathbf{d}
\end{gathered}
$$

as well as the following symbols

$$
\begin{gathered}
\mathbf{W}=y \boldsymbol{\zeta}+\boldsymbol{\omega}=y \boldsymbol{\zeta}-\mathbf{p}-\frac{1}{2} \mathbf{q} \theta \\
\mathbf{R}=\frac{\theta}{|\theta|} \mathbf{W}+\left(S \mathbf{v}+S \mathbf{d} \theta-\frac{1}{2} \mathbf{a} S|\theta||\mathbf{W}|\right)|\mathbf{W}|, \\
\mathbf{V}^{M} \equiv \mathbf{v}+\mathbf{d} \theta-\mathbf{a} S|\mathbf{W}||\theta|, \\
\mathbf{P} \equiv \mathbf{p}+\mathbf{q} \theta-\mathbf{d} S|\mathbf{W}||\theta|, \\
\mathbf{A} \equiv \mathbf{a}+\frac{\partial^{3}}{\partial \sigma \partial t^{2}} \mathbf{x}^{\prime}\left(\sigma_{0}, t\right) \theta-\frac{\partial^{3}}{\partial t^{3}} \mathbf{x}^{\prime}\left(\sigma_{0}, t\right) S|\mathbf{W}||\theta|, \\
\mathbf{D} \equiv \mathbf{d}+\frac{\partial^{3}}{\partial \sigma^{2} \partial t} \mathbf{x}^{\prime}\left(\sigma_{0}, t\right) \theta-\frac{\partial^{3}}{\partial t^{2} \partial \sigma} \mathbf{x}^{\prime}\left(\sigma_{0}, t\right) S|\mathbf{W}||\theta|, \\
\Delta_{2}=\left(C^{2}-v^{2}\right) S|\mathbf{W}|-2(\mathbf{d}, \mathbf{v}) S|\mathbf{W}| \theta+\frac{3}{2}(\mathbf{a}, \mathbf{v}) S^{2}|\mathbf{W}|^{2}|\theta| \\
-(\mathbf{W}, \mathbf{v}) \operatorname{sgn} \theta-(\mathbf{W}, \mathbf{d})|\theta|+(\mathbf{a}, \mathbf{W}) S|\mathbf{W}| \theta,
\end{gathered}
$$

needed according to (24) in order to express the derivatives at a point $(\sigma, \tau(\sigma))$ on the curve $\Gamma$ near $\sigma_{0}$. Then we can write

$$
\begin{gathered}
\mathbf{r}=\mathbf{R}|\theta|+\text { h.o.t. in }\left(\sigma-\sigma_{0}\right), \\
\frac{\partial}{\partial t^{\prime}} \mathbf{x}^{\prime}(\sigma, \tau(\sigma))=\mathbf{V}^{M}+\text { h.o.t., } \\
\frac{\partial}{\partial \sigma} \mathbf{x}^{\prime}(\sigma, \tau(\sigma))=\mathbf{P}+\text { h.o.t., } \\
\frac{\partial^{2}}{\partial \sigma \partial t} \mathbf{x}^{\prime}(\sigma, \tau(\sigma))=\mathbf{D}+\text { h.o.t., } \\
\frac{\partial^{2}}{\partial t^{2}} \mathbf{x}^{\prime}(\sigma, \tau(\sigma))=\mathbf{A}+\text { h.o.t., } \\
\Delta_{1}=\Delta_{2}|\theta|+\text { h.o.t., } \\
y \zeta+\boldsymbol{\omega}=\mathbf{W}+\text { h.o.t., }
\end{gathered}
$$

with $\mathbf{R}, \mathbf{V}, \mathbf{P}, \mathbf{D}$, and $\mathbf{A}$ given by (21).

C. Coefficients of the asymptotic expansion and the singular part of the stress. In order to obtain the coefficients of the asymptotic series which give the singular part of the displacement gradient (and from it the stress) we proceed by introducing 
expansions (19) with (20), (21), and (22) into the first integral of Eq. (12) which assumes the following form

$$
\int_{\alpha-\sigma_{0}}^{\beta-\sigma}\left[\left(\tilde{F}_{1}\right)_{m n}(\theta, y, c) \frac{1}{|\theta|}+\left(\tilde{F}_{2}\right)_{m n}(\theta, y, c) \frac{1}{\theta^{2}}\right] d \theta
$$

where $c$ may be $c_{1}$ or $c_{2}$, the longitudinal and shear wave speeds as before. To simplify the notation in the sequel we omit the indices $m n$ from the tensor functions $\tilde{F}_{i}$. The integral (23) can be written as the sum

$$
\int_{\alpha-\sigma_{0}}^{0} d \theta+\int_{0}^{\beta-\sigma_{0}} d \theta
$$

with the first integral written further

$$
\begin{aligned}
\int_{\alpha-\sigma_{0}}^{0} & {\left[\tilde{F}_{1}(\theta, y, c) \frac{1}{|\theta|}+\tilde{F}_{2}(\theta, y, c) \frac{1}{\theta^{2}}\right] d \theta } \\
= & \int_{0}^{\sigma_{0}-\alpha}\left[\tilde{F}_{1}(-\theta,-y, c) \frac{1}{\theta}+\tilde{F}_{2}(-\theta,-y, c) \frac{1}{\theta^{2}}\right] d \theta \\
& \equiv \int_{0}^{\sigma_{0}-\alpha}\left[\tilde{F}_{1}^{-}(\theta, y, c) \frac{1}{\theta}+\tilde{F}_{2}^{-}(\theta, y, c) \frac{1}{\theta^{2}}\right] d \theta
\end{aligned}
$$

so that the integral (23) assumes the form

$$
\int_{0}^{\beta-\sigma}\left(\frac{\tilde{F}_{1}}{\theta}+\frac{\tilde{F}_{2}}{\theta^{2}}\right) d \theta+\int_{0}^{\sigma-\alpha}\left(\frac{\tilde{F}_{1}^{-}}{\theta}+\frac{\tilde{F}_{2}^{-}}{\theta^{2}}\right) d \theta .
$$

Theorem 1 can be applied immediately to (24) to give the asymptotic expansion in $\varepsilon$

$$
\begin{aligned}
& \frac{1}{\varepsilon}\left[A_{0}^{\left(\tilde{F}_{1}\right)}+A_{0}^{\left(\tilde{F}_{1}^{-}\right)}+A_{1}^{\left(\dot{F}_{2}\right)}+A_{1}^{\left(\dot{F}_{2}^{-}\right)}\right] \\
& \quad+\ln \varepsilon\left[B_{1}^{\left(\tilde{F}_{1}\right)}+B_{1}^{\left(\tilde{F}_{1}^{-}\right)}+B_{2}^{\left(\tilde{F}_{2}\right)}+B_{2}^{\left(\tilde{F}_{2}^{-}\right)}\right]+\text {h.o.t. in } \varepsilon
\end{aligned}
$$

where the coefficients $A_{0}, A_{1}, B_{1}, B_{2}$ are according to their expressions in Theorem 1 . When evaluated for the functions of (24) they acquire the values

$$
B_{1}^{\left(\vec{F}_{1}\right)}=\left.F_{1}(\theta, y, c)\right|_{\theta=y=0^{+}}, \quad B_{1}^{\left(\tilde{F}_{1}\right)}=\left.F_{1}(\theta, y, c)\right|_{\theta=y=0^{+}}
$$

with

$$
\begin{gathered}
F_{1}(\theta, y, c)=\left(\frac{\varepsilon_{n j h} C_{i j k l} b_{i}}{4 \pi \rho}\right) \frac{R_{k} R_{m} R_{l}}{c^{3} S^{2}|\mathbf{W}|^{2} \Delta_{2}^{3}}\left(P_{h}(\mathbf{R}, \mathbf{A})+D_{h} \Delta_{2}\right) \\
-\left(\frac{b_{i} \varepsilon_{n r s}}{4 \pi}\right)\left[\frac{R_{i} R_{m}}{c S|\mathbf{W}| \Delta_{2}^{2}}\left(A_{r} P_{s}+V_{r}^{M} D_{s}\right)+\frac{R_{i} R_{m}}{c S|\mathbf{W}| \Delta_{2}^{3}}(\mathbf{R}, \mathbf{A}) V_{r}^{M} P_{s}\right] \\
B_{2}^{\left(\dot{F}_{2}^{-}\right)}=\left.\frac{\partial}{\partial \theta} F_{2}(\theta, y, C)\right|_{\theta=y=0^{+}}, \\
B_{2}^{\left(\hat{F}_{2}^{-}\right)}=\left.\frac{\partial}{\partial \theta} F_{2}(-\theta,-y, c)\right|_{\theta=y=0^{+}},
\end{gathered}
$$




$$
\begin{aligned}
F_{2}(\theta, y, c)= & \left(\frac{-\varepsilon_{n j h} C_{i j k l} b_{i}}{4 \pi \rho}\right)\left(\frac{P_{h}}{c^{3} S^{2}|\mathbf{W}|^{2} \Delta_{2}}\right) \\
& \times\left[\frac{1}{\Delta_{2}}\left(R_{l} R_{m} V_{k}^{M}+R_{l} R_{k} V_{m}^{M}+R_{k} R_{m} V_{l}^{M}\right)+\left(\delta_{k m} R_{l}+\delta_{m l} R_{k}+\delta_{k l} R_{m}\right)\right. \\
& \left.\quad-R_{l} R_{m} R_{k}\left(\frac{2}{S|\mathbf{W}| \Delta_{2}}+\frac{1}{\Delta_{2}^{2}}\left(c^{2}-\left(\mathbf{V}^{M} \mathbf{V}^{M}\right)\right)+\frac{3}{c^{2} S^{2}|\mathbf{W}|^{2}}\right)\right] \\
& +\left(\frac{b_{i} \varepsilon_{n r s}}{4 \pi}\right) P_{s} V_{r}^{M}\left[\frac{V_{i}^{M} R_{m}+R_{i} V_{m}^{M}}{c S|\mathbf{W}| \Delta_{2}^{2}}-\frac{3 R_{i} R_{m}}{c S^{2}|\mathbf{W}|^{2} \Delta_{2}^{2}}\right. \\
& \left.\quad \frac{\delta_{i m}}{c S \mathbf{W} \Delta_{2}}-\frac{R_{i} R_{m}\left(\mathbf{R}, \mathbf{V}^{M}\right)^{2}}{c^{3} S^{3}|\mathbf{W}|^{3} \Delta_{2}^{3}}+\frac{R_{i} R_{m}\left(\mathbf{V}^{M}, \mathbf{V}^{M}\right)}{c S|\mathbf{W}| \Delta_{2}^{3}}\right] .
\end{aligned}
$$

In order to evaluate $\left.F_{1}(\theta, y)\right|_{\theta=y=0}$ and $\left.F_{2}(\theta, y)\right|_{\theta=y=0}$ we need to know the $\lim _{\theta \rightarrow 0, y \rightarrow 0} S$ and $\lim _{\theta \rightarrow 0, y \rightarrow 0}(\partial / \partial \theta) S$. These limits may be easily obtained from the fundamental Eq. (4) and the definition of $S$ in Eq. (18) and are found to be

$$
\begin{aligned}
& S_{0} \equiv \lim _{\theta \rightarrow 0, y \rightarrow 0} S=\frac{1}{c^{2}-v^{2}}\{-\left(\mathbf{v}, \frac{\bar{p}}{|\mathbf{p}|}\right)+\sqrt{\left.c^{2}-v^{2}+\frac{(p, v)^{2}}{|\mathbf{p}|}\right\},} \\
&\left(\frac{\partial S}{\partial \theta}\right)_{0}=\lim _{\substack{\theta \rightarrow 0 \\
y \rightarrow 0}} \frac{\partial S}{\partial \theta}=\frac{S_{0}}{2\left(c^{2}-v^{2}\right)}\left\{-\frac{(\mathbf{v}, \mathbf{q})}{|\mathbf{p}|}+\frac{(\mathbf{v}, \mathbf{p})(\mathbf{p}, \mathbf{q})}{|\mathbf{p}|^{3}}-2\left(\frac{\mathbf{p}}{|\mathbf{p}|}, \mathbf{d}\right)\right. \\
&\left.+[2(\mathbf{v}, \mathbf{d})+(\mathbf{a}, \mathbf{p})] S_{0}-(\mathbf{v}, \mathbf{a})|\mathbf{p}| S_{0}^{2}\right\} .
\end{aligned}
$$

The rest of the coefficients in (30) are

$$
\begin{gathered}
A_{0}^{\left(\tilde{F}_{1}\right)}=A_{0}^{\left(\tilde{F}_{1}^{-}\right)}=0, \\
A_{1}^{\left(\tilde{F}_{2}\right)}=\int_{0}^{\infty} F_{2}\left(0^{+}, \xi, c\right) d \xi \\
A_{1}^{\left(\tilde{F}_{2}^{-}\right)}=\int_{0}^{\infty} F_{2}\left(0^{-},-\xi, c\right) d \xi,
\end{gathered}
$$

where for the last integral in (32) we further need to know

$$
S_{1} \equiv \lim _{\theta \rightarrow 0} S=\frac{1}{c^{2}-v^{2}}\left\{\frac{(\theta \mathbf{W}, \mathbf{v})}{|\theta \mathbf{W}|}+\sqrt{\left(\mathbf{v}, \frac{\mathbf{W}}{|\mathbf{W}|}\right)^{2}+c^{2}-\mathbf{v}^{2}}\right\} .
$$

This completes the determination of the coefficients of Eq. (24) which only refers to the first integral of (12). Before proceeding in the evaluation of the second term of (12) which involves the step functions in the Green's function, and hence double integrals, we give the coefficients in the asymptotic expansion of (14) which gives the contribution to the strain from the last term of the Green's function in Eq. (13). The procedure for the asymptotic treatment of the integral (14) is exactly analogous to the one just completed and we give it succinctly below

$$
\int_{0}^{\beta-\sigma}\left(\frac{\tilde{F}_{3}}{\theta}+\frac{\tilde{F}_{4}}{\theta^{2}}\right) d \theta+\int_{0}^{\sigma-\alpha}\left(\frac{\tilde{F}_{3}^{-}}{\theta}+\frac{\tilde{F}_{4}^{-}}{\theta^{2}}\right) d \theta
$$


which in the asymptotic expansion give

$$
\begin{aligned}
& \frac{1}{\varepsilon}\left[A_{0}^{\left(\tilde{F}_{3}\right)}+A_{0}^{\left(\tilde{F}_{3}^{-}\right)}+A_{1}^{\left(\tilde{F}_{4}\right)}+A_{1}^{\left(\tilde{F}_{4}^{-}\right)}\right] \\
& \quad+\ln \varepsilon\left[B_{1}^{\left(\tilde{F}_{3}\right)}+B_{1}^{\left(\tilde{F}_{3}^{-}\right)}+B_{2}^{\left(\tilde{F}_{3}\right)}+B_{2}^{\left(\tilde{F}_{3}^{-}\right)}\right]+\text {h.o.t. in } \varepsilon
\end{aligned}
$$

with

$$
\begin{aligned}
B_{1}^{\left(\tilde{F}_{3}\right)} & =\left.F_{3}\left(\theta, y, c_{2}\right)\right|_{\theta=y=0^{+}}, \\
B_{1}^{\left(\tilde{F}_{3}^{-}\right)} & =\left.F_{3}\left(-\theta,-y, c_{2}\right)\right|_{\theta=y=0^{+}}, \\
B_{2}^{\left(\tilde{F}_{4}\right)} & =\left.\frac{\partial}{\partial \theta} F_{4}\left(\theta, y, c_{2}\right)\right|_{\theta=y=0^{+}}, \\
B_{2}^{\left(\tilde{F}_{4}^{-}\right)} & =\left.\frac{\partial}{\partial \theta} F_{4}\left(-\theta,-y, c_{2}\right)\right|_{\theta=y=0^{+}}, \\
A_{0}^{\left(\tilde{F}_{3}\right)} & =A_{0}^{\left(\tilde{F}_{3}^{-}\right)}=0, \\
A_{1}^{\left(\tilde{F}_{3}\right)} & =\int_{0}^{\infty} F_{4}\left(0^{+}, \xi, c_{2}\right) d \xi, \\
A_{1}^{\left(\tilde{F}_{3}^{-}\right)} & =\int_{0}^{\infty} F_{4}\left(0^{+},-\xi, c_{2}\right) d \xi,
\end{aligned}
$$

with

$$
\begin{aligned}
F_{3}\left(\theta, y ; c_{2}\right)= & \left(\frac{\varepsilon_{n j h} C_{i j k l} b_{i}}{4 \pi \rho c_{2}}\right)\left[\frac{-R_{l} D_{h}}{\Delta_{2}^{2}}+\frac{-P_{h} R_{l}(\mathbf{R}, \mathbf{A})}{\Delta_{2}^{3}}\right] \delta_{k m} \\
& \left.+\left(\frac{b_{i} \varepsilon_{n r s}}{4 \pi c_{2}}\right) \frac{c_{2}^{2} S \mathbf{W}}{\Delta_{2}^{3}}\left[\left(D_{s} V_{r}^{M}+P_{s} A_{r}\right) \Delta_{2}+P_{s} V_{r}(\mathbf{R}, \mathbf{A})\right)\right] \delta_{i m} \\
F_{4}\left(\theta, y ; c_{2}\right)= & \left(\frac{\varepsilon_{n j h} C_{i j k l} b_{i}}{4 \pi \rho c_{2}}\right)\left[\frac{P_{h} V_{l}^{M}}{\Delta_{2}^{2}}-\frac{R_{l} P_{h}}{\Delta_{2}^{3}}\left(c_{2}^{2}-\left(\mathbf{V}^{M}, \mathbf{V}^{M}\right)\right)\right] \delta_{k m} \\
& +\left(\frac{b_{i} \varepsilon_{n r s} c_{2}}{4 \pi}\right)\left[\frac{\left(\mathbf{R}, \mathbf{V}^{M}\right)-c_{2}^{2} S \mathbf{W}(\mathbf{V}, \mathbf{V})}{\Delta_{2}^{3}}\right] P_{s} V_{r}^{M} \delta_{i m} .
\end{aligned}
$$

We last have to evaluate asymptotically the double integral in the last term of Eq. (12), i.e., an integral of the form

$$
\int_{\alpha}^{\beta} d \sigma \int_{\tau}^{t} E\left(\theta, t, y, t^{\prime}\right) d t^{\prime}
$$

The inner integral has the property that near the singular point $\left(\sigma_{0}, t\right)$ the upper and lower limits approach each other. In order to avoid this and obtain an integral from 0 to a finite upper limit we change the variable of integration: $t-t^{\prime}=|\theta| u$, so that

$$
\int_{\tau}^{t} E\left(\theta, t, y, t^{\prime}\right) d t^{\prime}=\int_{0}^{S|\mathbf{W}|}|\theta| E_{1}(\theta, t, y, u) d u=\int_{0}^{S}|\theta||\mathbf{W}| E_{1}(\theta, t, y,|\mathbf{W}| u) d u
$$

where $\lim _{\theta \rightarrow 0, y \rightarrow 0} S$ is $O(1)$.

In order to obtain $E_{1}$ we need to express every element under this double integral (39) in terms of $\theta$ and $u$, e.g.,

$$
\mathbf{r}=\varepsilon \zeta+\theta \boldsymbol{\omega}+\mathbf{x}^{\prime}(\sigma, t)-\mathbf{x}^{\prime}\left(\sigma, t^{\prime}\right)
$$


with

$$
\begin{aligned}
& \frac{\partial}{\partial t} \mathbf{x}^{\prime}(\sigma, t)=\frac{\partial}{\partial t} \mathbf{x}^{\prime}\left(\sigma_{0}, \tau\right)+\frac{\partial^{2}}{\partial t \partial \sigma} \mathbf{x}^{\prime}\left(\sigma_{0}, t\right) \theta+\text { h.o.t. in } \theta \\
& =\mathbf{v}+\mathbf{d} \theta+\text { h.o.t. of } \theta, \\
& \frac{\partial^{2}}{\partial t^{2}} \mathbf{x}^{\prime}(\sigma, t)=\mathbf{a}+\text { h.o.t. of } \theta, \\
& \mathbf{x}^{\prime}(\sigma, t)-\mathbf{x}^{\prime}\left(\sigma, t^{\prime}\right)=(\mathbf{v}+\mathbf{d} \theta)\left(t-t^{\prime}\right)-\frac{1}{2} \mathbf{a}\left(t-t^{\prime}\right)^{2}+\text { h.o.t. }
\end{aligned}
$$

so that

$$
\mathbf{r}=\varepsilon \zeta+\theta \omega+\left[\mathbf{v}+\mathbf{d} \theta-\frac{1}{2} \mathbf{a}|\theta| u\right]|\theta| u+\text { h.o.t. }=|\theta| \mathbf{R}^{\prime}(\theta, y, u)+\text { h.o.t. }
$$

with

$$
\mathbf{R}^{\prime}=\frac{\theta}{|\theta|} \mathbf{W}+\left(\mathbf{v}+\mathbf{d} \theta-\frac{1}{2} \mathbf{a}|\theta| u\right) u
$$

and

$$
\bar{r}=|\theta|\left(\mathbf{R}^{\prime}, \mathbf{R}^{\prime}\right)^{1 / 2}+\text { h.o.t. }
$$

It is easy to see, if we define in an analogous way as (21)

$$
\begin{gathered}
\mathbf{V}^{\prime} \equiv \mathbf{v}+\mathbf{d} \theta-\mathbf{a}|\theta| u, \quad \text { then } \frac{\partial}{\partial t^{\prime}} \mathbf{x}^{\prime}\left(\sigma, t^{\prime}\right)=\mathbf{V}^{\prime}+\text { h.o.t., } \\
\mathbf{P}^{\prime} \equiv \mathbf{p}+\mathbf{q} \theta-\mathbf{d}|\theta| u, \quad \text { then } \frac{\partial}{\partial \sigma} \mathbf{x}^{\prime}\left(\sigma, t^{\prime}\right)=\mathbf{p}^{\prime}+\text { h.o.t., } \\
\mathbf{A}^{\prime} \equiv \mathbf{a}+\frac{\partial^{3}}{\partial \sigma \partial t^{2}} \mathbf{x}^{\prime}\left(\sigma_{0}, t\right) \theta-\frac{\partial^{3}}{\partial t^{3}} \mathbf{x}^{\prime}\left(\sigma_{0}, t\right)|\theta| u \\
\text { then } \frac{\partial^{2}}{\partial t^{2}} \mathbf{x}^{\prime}\left(\sigma, t^{\prime}\right)=\mathbf{A}^{\prime}+\text { h.o.t. } \\
\mathbf{D}^{\prime} \equiv \mathbf{d}^{\prime}+\frac{\partial^{3}}{\partial \sigma^{2} \partial t} \mathbf{x}^{\prime}\left(\sigma_{0} t\right) \theta-\frac{\partial^{3}}{\partial \sigma \partial t^{2}} \mathbf{x}^{\prime}\left(\sigma_{0}, t\right)|\theta| u \\
\text { then } \frac{\partial^{2}}{\partial \sigma \partial t} \mathbf{x}^{\prime}\left(\sigma, t^{\prime}\right)=\mathbf{D}^{\prime}+\text { h.o.t. }
\end{gathered}
$$

Therefore the integral (39) becomes

$$
\int_{0}^{\beta-\sigma_{0}} \frac{d \theta}{\theta^{2}} \tilde{F}_{5}(\theta, y, t, c)+\int_{0}^{\sigma_{0}-\alpha} \frac{d \theta}{\theta^{2}} \tilde{F}_{5}^{-}(\theta, y, t, c)
$$

which in the asymptotic expansion give

$$
\frac{1}{\varepsilon}\left[A_{1}^{\left(\tilde{F}_{s}\right)}+A_{1}^{\left(\tilde{F}_{s}^{-}\right)}\right]+\ln \varepsilon\left[B_{2}^{\left(\tilde{F}_{s}\right)}+B_{2}^{\left(\tilde{F}_{s}^{-}\right)}\right]
$$

with

$$
\begin{gathered}
A_{1}^{\left(\tilde{F}_{5}\right)}=\int_{0}^{\infty} d \xi F_{5}\left(0^{+}, \xi, c\right), \\
A_{1}^{\left(\tilde{F}_{5}^{-}\right)}=\int_{0}^{\infty} d \xi F_{5}\left(0^{-},-\xi, c\right), \\
B_{2}^{\left(\tilde{F}_{5}\right)}=\left.\frac{\partial}{\partial \theta} F_{5}(\theta, y, c)\right|_{\theta=y=0^{+}}, \quad B_{2}^{\left(\tilde{F}_{5}^{-}\right)}=\left.\frac{\partial}{\partial \theta} F_{5}(-\theta,-y, c)\right|_{\theta=y=0^{+}}
\end{gathered}
$$


with

$$
\begin{aligned}
F_{5}(\theta, y, c)=\int_{0}^{S}|\mathbf{W}| d u\{ & \left(\frac{\varepsilon_{n j h} C_{i j k l} b_{i}}{4 \pi \rho}\right) P_{h}^{\prime} u|\mathbf{W}| \\
& \times\left[\frac{3\left(\delta_{m k} R_{l}^{\prime}+\delta_{k l} R_{m}^{\prime}+\delta_{m l} R_{k}^{\prime}\right)}{R^{\prime 5}}-\frac{15 R_{l}^{\prime} R_{m}^{\prime} R_{k}^{\prime}}{R^{\prime 7}}\right] \\
& \left.+\left(\frac{b_{i} \varepsilon_{n r s}}{4 \pi}\right) V_{r}^{\prime} P_{s}^{\prime}\left(\frac{3 R_{i}^{\prime} R_{m}^{\prime}}{R^{\prime 5}}-\frac{\delta_{i m}}{R^{\prime 3}}\right)\right\} .
\end{aligned}
$$

The asymptotic expansion (42) completes the expansion of the double integral in the solution arising from the Heaviside step function in the Green's function. The final result that gives the displacement gradient $V_{m, n}$ at a point near the moving loop consists, according to (12), of combinations of (25), (35), and (42) and assumes the following expression

$$
\begin{array}{r}
\frac{1}{\varepsilon} \int_{0}^{\infty}\left[F_{2}\left(0^{+}, \xi, c_{2}\right)+F_{2}\left(0^{-},-\xi, c_{2}\right)-F_{2}\left(0^{+}, \xi, c_{1}\right)-F_{2}\left(0^{-},-\xi, c_{1}\right)\right. \\
+F_{5}\left(0^{+}, \xi, c_{2}\right)+F_{5}\left(0^{-},-\xi, c_{2}\right)-F_{5}\left(0^{+}, \xi, c_{1}\right)-F_{5}\left(0^{-},-\xi, c_{1}\right) \\
\left.+F_{4}\left(0^{+}, \xi, c_{2}\right)+F_{4}\left(0^{-},-\xi, c_{2}\right)\right] d \xi \\
+\ln \varepsilon\left\{F_{1}\left(0^{+}, 0^{+}, c_{2}\right)+F_{1}\left(0^{-}, 0^{-}, c_{2}\right)-F_{1}\left(0^{+}, 0^{+}, c_{1}\right)\right. \\
-F_{1}\left(0^{-}, 0^{-}, c_{1}\right)+F_{3}\left(0^{+}, 0^{+}, c_{2}\right)+F_{3}\left(0^{-}, 0^{-}, c_{2}\right) \\
+\frac{\partial}{\partial \theta}\left[F_{2}\left(\theta, 0^{+}, c_{2}\right)+F_{2}\left(-\theta, 0^{-}, c_{2}\right)-F_{2}\left(\theta, 0^{+}, c_{1}\right)+F_{2}\left(-\theta, 0^{-}, c_{1}\right)\right. \\
+F_{4}\left(\theta, 0^{+}, c_{2}\right)+F_{4}\left(-\theta, 0^{-}, c_{2}\right)+F_{5}\left(\theta, 0^{+}, c_{2}\right)+F_{5}\left(-\theta, 0^{-}, c_{2}\right) \\
\left.\left.-F_{5}\left(\theta, 0^{+}, c_{1}\right)-F_{5}\left(\theta, 0^{-}, c_{1}\right)\right]\right\}\left.\right|_{\theta=0^{+}}+\text {h.o.t. in } \varepsilon
\end{array}
$$

where the indices $m n$ in the tensor functions $\left(F_{i}\right)_{m n}$ have been omitted.

Example. As an example we want to retrieve from the loop solution the solution for the near field of an infinitely long screw dislocation. As shown in Fig. 2 the loop lies on the $x_{1}-x_{2}$ plane and as its radius tends to infinity it approximates an infinitely long screw dislocation tangent to the loop at the point $\Sigma$. The straight dislocation line is assumed to move with constant acceleration $a$, so that $\mathbf{x}^{\prime}=\left(\frac{1}{2} a t^{\prime 2} H\left(t^{\prime}\right), x_{2}^{\prime}, 0\right)$. For near-field evaluation the field point is $\mathbf{x}=\left(\frac{1}{2} a t^{2}+\varepsilon \cos \delta, 0, \varepsilon \sin \delta\right)$. For the displacement gradient $U_{2,3}$ we have from (2)

$$
\begin{aligned}
U_{2,3}= & \int_{-\infty}^{t} d t^{\prime} \int_{-\infty}^{\infty} b C_{1212}\left(G_{12,2}+G_{22,1}\right) d x_{2}^{\prime} \\
& +\int_{-\infty}^{t} d t^{\prime} \int_{-\infty}^{\infty} b_{2} \rho V_{1} \dot{G}_{22} d x_{2}^{\prime} .
\end{aligned}
$$

From symmetry arguments, as presented for instance in Achenbach [1], p. 107, for anti-plane symmetry - as is the case for the infinitely long screw dislocation here(45) becomes

$$
U_{2,3}=\int_{-\infty}^{+} d t^{\prime} \int_{-\infty}^{\infty}\left[b_{2} C_{1212} G_{22,1}+\rho b_{2} V_{1} \dot{G}_{22}\right] d x_{2}^{\prime}
$$




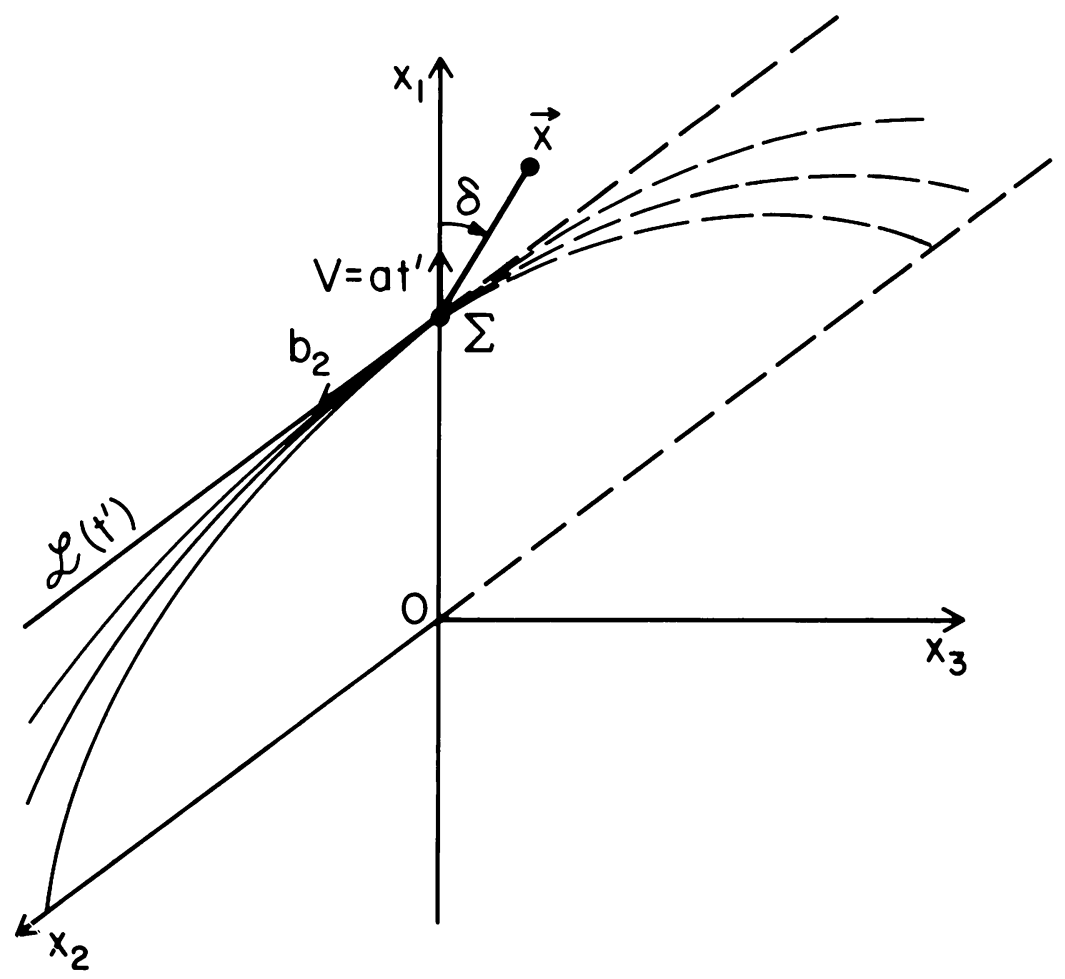

FIG. 2

where in $G_{22}$ only the term $\delta\left(\bar{t}-\bar{r} / c_{2}\right) / c_{2}^{2} \bar{r}$ contributes.

In order to check the solution (44) it was indeed obtained that the other terms of the Green's function contribute zero to the solution. The time integral in (46) may be further split into two parts, the first one being

$$
\int_{-\infty}^{t} d t^{\prime} \int_{-\infty}^{\infty}\left[b_{2} c_{1212} G_{22,1}\right] d x_{2}^{\prime}=-\frac{b_{2}}{2 \pi} \frac{1}{x}
$$

according to Appendix A. While for the second one we have according to (44) and with only $F_{3}, F_{4} \neq 0$,

$$
\begin{aligned}
U_{2,3}= & \left.\frac{1}{\varepsilon} \int_{0}^{\infty}\left[F_{4}\left(0, \xi, c_{2}\right)+F_{4}\left(-\theta,-\xi, c_{2}\right)\right]\right|_{\theta=0^{+}} d \xi \\
& +\ln \varepsilon\left\{F_{3}\left(\theta^{+}, y, c_{2}\right)+F_{3}\left(-\theta,-y, c_{2}\right)\right. \\
& \left.\quad+\frac{\partial}{\partial \theta}\left[F_{4}\left(\theta, y, c_{2}\right)+F_{4}\left(-\theta,-y, c_{2}\right)\right]\right\}\left.\right|_{\theta=y=0^{+}}
\end{aligned}
$$


with $F_{3}, F_{4}$ given by (37) and (38) respectively with the indices $n=3, m=i=h=$ $k=s=2, j=l=r=1$ so that

$$
\begin{aligned}
F_{3}\left(\theta, y, c_{3}\right)= & \frac{C_{1212} b_{2}}{4 \pi \rho c_{2}}\left[-\frac{R_{1} D_{2}}{\Delta_{2}^{2}}+\frac{-P_{2} R_{1}(\mathbf{R}, \mathbf{A})}{\Delta_{2}^{3}}\right] \\
& +\frac{b_{2}}{4 \pi c_{2}} \frac{c_{2}^{2} S|\mathbf{W}|}{\Delta_{2}^{3}}\left[\left(D_{2} V_{1}^{M}+P_{2} A_{1}\right) \Delta_{2}+P_{2} V_{1}^{M}(\mathbf{R}, \mathbf{A})\right], \\
F_{4}\left(\theta, y, c_{2}\right)= & \frac{C_{1212} b_{2}}{4 \pi \rho c_{2}}\left[\frac{P_{2} V_{1}^{M}}{\Delta_{2}^{2}}-\frac{R_{1} P_{2}}{\Delta^{3}}\left(c_{2}^{2}-\left(\mathbf{V}^{M}, \mathbf{V}^{M}\right)\right)\right] \\
& +\frac{b_{2}}{4 \pi c_{2}}\left[\frac{c_{2}^{2}\left(\mathbf{R}, \mathbf{V}^{M}\right)}{\Delta_{2}^{3}}-\frac{c_{2}^{2} S|\mathbf{W}|\left(\mathbf{V}^{M}, \mathbf{V}^{M}\right)}{\Delta_{2}^{3}}\right] P_{2} V_{1}^{M},
\end{aligned}
$$

where the variables in (49) and (50) for this specific motion and field point take the values

$$
\begin{gathered}
\mathbf{V}=(v, 0,0), \\
\mathbf{a}=(a, 0,0), \\
\mathbf{p}=(0,1,0), \\
\boldsymbol{\zeta}=(\cos \delta, 0, \sin \delta), \\
\mathbf{W}=(y \cos \delta,-1, y \sin \delta), \\
\mathbf{W}=\sqrt{1+y^{2}}, \\
\mathbf{V}^{M}=\left(\mathbf{v}-a S|\theta| \sqrt{1+y^{2}}, 0,0\right), \\
\mathbf{P}=(0,1,0), \\
\mathbf{A}=(a, 0,0), \\
\mathbf{D}=0, \\
\mathbf{R}=\frac{\theta}{|\theta|} \mathbf{W}+\left(S \mathbf{v}-\frac{1}{2} \mathbf{a} S^{2}|\theta||\mathbf{W}|\right)|\mathbf{W}| \\
=\left(\frac{\theta}{|\theta|} y \cos \delta+\left(s \mathbf{v}-\frac{1}{2} a s^{2}|\theta| \sqrt{1+y^{2}}\right) \sqrt{1+y^{2}}, \frac{-\theta}{|\theta|}, 0\right) \\
\Delta_{2}=\left(c_{2}^{2}-v^{2}\right) S \sqrt{1+y^{2}}+\frac{3}{2} a v s^{2} \sqrt{1+y^{2}}|\theta|-y \cos \delta v \operatorname{sgn}(\theta)+a y \cos \delta S \theta \sqrt{1+y}, \\
S_{0}=\left(c_{2}-v^{2}\right)-1 / 2
\end{gathered}
$$$$
S_{0}=\left(c_{2}^{2}-v^{2}\right)^{-1 / 2} \text {, }
$$$$
\left(\frac{\partial s}{\partial \theta}\right)_{0}=\frac{-v a}{2\left(c_{2}^{2}-v^{2}\right)^{2}},
$$$$
S_{1}=\frac{1}{c_{2}^{2}-v^{2}}\left\{\frac{\theta y v \cos \delta}{|\theta| \sqrt{1+y^{2}}}+\sqrt{\frac{y^{2} v^{2} \cos ^{2} \delta}{\left(1+y^{2}\right)}+\left(c_{2}^{2}-\nu^{2}\right)}\right\} \text {, }
$$$$
\left.\Delta_{20} \equiv \Delta_{2}\right|_{\theta=y=0}=\left(c_{2}^{2}-v^{2}\right)^{1 / 2},
$$$$
\left.\Delta_{2}\right|_{\theta=0}=\left(c_{2}^{2}-v^{2}\right) S_{1} \sqrt{1+y^{2}}-y v \cos \delta
$$$$
=\left[\left(c_{2}^{2}-v^{2}\right)\left(1+y^{2}\right)+y^{2} v^{2} \cos ^{2} \delta\right]^{1 / 2},
$$ 


$$
\left(\frac{\partial \Delta_{2}}{\partial \theta}\right)_{0}=a \frac{v}{\left(c_{2}^{2}-v^{2}\right)}
$$

so that we can obtain the functions $F_{4}\left(0, y, c_{2}\right)$,

$$
\begin{aligned}
F_{4}\left(0^{+}, y, c_{2}\right)= & F_{4}\left(0^{-},-y, c_{2}\right)=\frac{b_{2} c_{2}}{4 \pi}\left[\frac{v}{\Delta_{2}^{2}}-\frac{\left(c^{2}-v^{2}\right)\left(y \cos \delta+S_{1} v \sqrt{1+y^{2}}\right)}{\left.\Delta_{2}^{3}\right|_{\theta=0}}\right] \\
& +\frac{b_{2} c_{2}}{4 \pi}\left[\frac{v^{2}\left(y \cos \delta+S_{1} v \sqrt{1+y^{2}}\right)-S_{1} v^{3} \sqrt{1+y^{2}}}{\left.\Delta_{2}^{3}\right|_{\theta=0}}\right] \\
= & -\frac{b_{2} c_{2}}{4 \pi} \frac{\left(c_{2}^{2}-v^{2}\right) y \cos \delta}{\left.\Delta_{2}^{3}\right|_{\theta=0}}
\end{aligned}
$$

and the coefficient of $1 / \varepsilon$ in the asymptotic expansion (48) acquires the value

$$
-2 \int_{0}^{\infty} \frac{b_{2} c_{2}}{4 \pi} \frac{\left(c_{2}^{2}-v^{2}\right) \xi \cos \delta d \xi}{\left[\left(c_{2}^{2}-v^{2}\right)\left(1+\xi^{2}\right)+\xi^{2} v^{2} \cos ^{2} \delta\right]^{3 / 2}}=-\frac{b_{2} c_{2}}{2 \pi} \frac{\cos \delta \sqrt{c_{2}^{2}-v^{2}}}{\left(c_{2}^{2}-v^{2} \sin ^{2} \delta\right)}
$$

however we have from (49) and (50)

$$
\begin{aligned}
F_{3}\left(0^{+}, 0^{+} c_{2}\right)= & F_{3}\left(0^{-}, 0^{-}, c_{2}\right) \\
= & \left(\frac{b_{2} c_{2}}{4 \pi}\right)\left(\frac{-a v^{2}}{\left(c_{2}^{2}-v^{2}\right)^{5 / 2}}\right)+\left(\frac{b_{2}}{4 \pi c_{2}}\right)\left[\frac{c_{2}^{2} a v^{2}}{\left(c_{2}^{2}-v^{2}\right)^{3 / 2}}+\frac{c_{2}^{2} a v^{2}}{\left(c_{2}^{2}-v^{2}\right)^{5 / 2}}\right] \\
=\left(\frac{b_{2}}{4 \pi}\right) \frac{a c_{2}}{\left(c_{2}^{2}-v^{2}\right)^{3 / 2}} & \\
F_{4}\left(\theta, 0^{+}, c_{2}\right)=F_{4}\left(\theta, 0^{-}, c_{2}\right) & =\left(\frac{b_{2} c_{2}}{4 \pi}\right) \frac{\left(-\frac{1}{2} a s^{2} \theta\right)\left(c^{2}-(v-a S \theta)^{2}\right)}{\Delta_{2}^{3}}, \\
\left.\frac{\partial}{\partial \theta} F_{4}\left(\theta, 0, c_{2}\right)\right|_{\theta=0} & =\left.\frac{\partial}{\partial \theta} F_{4}\left(-\theta, 0, c_{2}\right)\right|_{\theta=0} \\
& =\left(\frac{b_{2} c_{2}}{4 \pi}\right) \frac{\left(-\frac{1}{2} a S^{2}\right)\left(c^{2}-v^{2}\right)}{\Delta_{2}^{3}} \\
& =\frac{-1 / 2 a c_{2}}{\left(c^{2}-v^{2}\right)^{3 / 2}}\left(\frac{b_{2}}{4 \pi}\right)
\end{aligned}
$$

so that the coefficient of $\ln \varepsilon$ in (48) obtains the value

$$
\left(\frac{b_{2}}{4 \pi}\right) \frac{a}{c_{2}^{2}\left(1-v^{2} / c_{2}^{2}\right)^{3 / 2}}
$$

Thus the total expression for the asymptotic expansion of $U_{2,3}$ near the current position of the screw dislocation moving with constant acceleration from rest is

$$
U_{2,3}=\frac{-b_{2}}{2 \pi} \frac{\cos \delta \sqrt{1-v^{2} / c_{2}^{2}}}{\left(1-\left(v^{2} / c_{2}^{2}\right) \sin ^{2} \delta\right)} \frac{1}{\varepsilon}+\frac{b_{2}}{4 \pi} \frac{a}{c_{2}^{2}\left(1-\left(v^{2} / c_{2}^{2}\right)\right)^{3 / 2}} \ln \varepsilon+\text { h.o.t. }
$$

The $1 / \varepsilon$ term agrees with the steady state solution with the instantaneous velocity $v=$ at and the $\ln \varepsilon$ coefficient agrees with the solution obtained by Callias and Markenscoff [3]. 
Conclusions. The near field of a loop moving arbitrarily has been analyzed and the coefficients of the $\frac{1}{\varepsilon}$ and $\ln \varepsilon$ singular terms obtained. The case of a straight screw dislocation is retrieved. The expressions for the general shape loop are lengthy; in a subsequent paper by Markenscoff and $\mathrm{Ni}$ [17] the geometric features of this solution are explored and examples are presented for a circular loop.

Acknowledgment. One of the authors, Luqun $\mathrm{Ni}$, was supported in part by the Center of Excellence for Advanced Materials at the University of California, San Diego.

Appendix A. For a screw dislocation with $b_{2} \neq 0$ being at rest until time $t^{\prime}=0$ and then starting to move with a constant velocity $V$, we have according to Eq. (2) and for $\mathbf{x}^{\prime}=\left(V H\left(t^{\prime}\right) t^{\prime}, x_{2}^{\prime}, 0\right) \mathbf{x}=\left(x_{1}, 0,0\right)$

$$
\begin{aligned}
V_{2,3}\left(x_{1}, 0,0, t\right)= & \int_{-\infty}^{t} d t^{\prime} \int_{-\infty}^{\infty} b_{2} G_{22,1} C_{1212} d x_{2}^{\prime}+\int_{0}^{t} d t^{\prime} \int_{-\infty}^{\infty} b_{2} \rho V \dot{G}_{22} d x_{2}^{\prime} \\
= & \int_{-\infty}^{0} d t^{\prime} \int_{-\infty}^{\infty} b_{2} G_{22,1} C_{1212} d x_{2}^{\prime}+\int_{0}^{t} d t^{\prime} \int_{-\infty}^{\infty} b_{2} C_{1212} G_{22,1} d x_{2}^{\prime} \\
& +\int_{0}^{t} \int_{-\infty}^{\infty} b_{2} \rho V \dot{G}_{22} d x_{2}^{\prime} \\
\equiv & \mathrm{I}+\mathrm{II}+\mathrm{III} .
\end{aligned}
$$

Integral I is performed to give

$$
\begin{aligned}
\mathbf{I} & =\frac{b_{2}}{4 \pi} \int_{-\infty}^{0} d t^{\prime} \frac{\partial}{\partial x_{1}}\left[\frac{2 c_{2} H\left(c_{2}^{2}\left(t-t^{\prime}\right)^{2}-x_{1}^{2}\right)}{\sqrt{c_{2}^{2}\left(t-t^{\prime}\right)^{2}-x_{1}^{2}}}\right] \\
& =\frac{b_{2} c_{2}}{2 \pi} \int_{-\infty}^{0} d t^{\prime}\left(\frac{x_{1}}{c^{2}\left(t-t^{\prime}\right)}\right) \frac{\partial}{\partial t^{\prime}}\left[\frac{H\left(c^{2}\left(t-t^{\prime}\right)^{2}-x_{1}^{2}\right)}{\sqrt{c_{2}^{2}\left(t-t^{\prime}\right)^{2}-x_{1}^{2}}}\right] \\
& =\frac{b_{2}}{2 \pi} \frac{x_{1}}{c_{2}}\left[\left.\frac{1}{\left(t-t^{\prime}\right)} \frac{H\left(c^{2} \bar{t}^{2}-x_{1}^{2}\right)}{\sqrt{c^{2}\left(t-t^{\prime}\right)^{2}-x_{1}^{2}}}\right|_{-\infty} ^{0}-\int_{-\infty}^{0} \frac{1}{\left(t-t^{\prime}\right)^{2}} \frac{H\left(c^{2}\left(t-t^{\prime}\right)^{2}-x_{1}^{2}\right)}{\sqrt{c^{2}\left(t-t^{\prime}\right)^{2}-x_{1}^{2}}} d t^{\prime}\right]
\end{aligned}
$$

in which case, for $c t<\left|x_{1}\right|$ it yields

$$
-\frac{b_{2} x_{1}}{2 \pi c_{2}} \int_{-\infty}^{0} \frac{1}{\left(t-t^{\prime}\right)^{2}} \frac{H\left(c^{2}\left(t-t^{\prime}\right)^{2}-x_{1}^{2}\right)}{\sqrt{c^{2}\left(t-t^{\prime}\right)^{2}-x_{1}^{2}}} d t^{\prime}=\frac{-b_{2}}{2 \pi} \frac{1}{x_{1}}
$$


with the contribution, which corresponds to the static solution, coming from $t^{\prime}=-\infty$. For $c t>\left|x_{1}\right|$ the integral yields

$$
\begin{aligned}
\mathrm{I} & =\frac{b_{2}}{2 \pi} \frac{x_{1}}{c_{2}}\left[\frac{1}{t \sqrt{c^{2} t^{2}-x_{1}^{2}}}-\int_{-\infty}^{0} \frac{1}{\left(t-t^{\prime}\right)^{2}} \frac{d t^{\prime}}{\sqrt{c^{2}\left(t-t^{\prime}\right)^{2}-x_{1}^{2}}}\right] \\
& =\frac{b_{2}}{2 \pi} \frac{x_{1}}{c_{2}}\left[\frac{1}{t \sqrt{c^{2} t^{2}-x_{1}^{2}}}+\frac{\sqrt{c^{2} t^{2}-x_{1}^{2}}}{x_{1}^{2} t^{2}}-\frac{c}{x_{1}^{2}}\right] \\
& =\frac{b_{2}}{2 \pi}\left[\frac{1}{x_{1}}+\frac{c t}{x_{1} \sqrt{c^{2} t^{2}-x^{2}}}\right]
\end{aligned}
$$

where in the last expression the contributions are due to $t^{\prime}=-\infty$ (static part) and $t^{\prime}=0$ respectively.

From the integrals II and III we obtain

$$
\begin{gathered}
0, \text { for } c t<\left|x_{1}\right| \\
-\frac{b_{2}}{2 \pi} \frac{c^{2} t-x_{1} v}{c\left(x_{1}-v t\right) \sqrt{c^{2} t^{2}-x_{1}^{2}}}, \text { for } c t>\left|x_{1}\right|
\end{gathered}
$$

so that the final result is

$$
U_{2,3}= \begin{cases}\frac{b_{2}}{2 \pi} \frac{1}{x_{1}}, & \text { for } c t<\left|x_{1}\right| \\ -\frac{b_{2}}{2 \pi} \frac{1}{x_{1}}-\frac{v \sqrt{c^{2} t^{2}-x_{1}^{2}}}{x_{1} c\left(x_{1}-v t\right)}, & \text { for } c t>\left|x_{1}\right|\end{cases}
$$

which is in agreement with Eq. (17) of Markenscoff [12] obtained by a different approach.

\section{REFERENCES}

[1] J. D. Achenbach, Wave Propagation in Elastic Solids, North Holland, Amsterdam-London, 1973

[2] R. Burridge and L. Knopoff, Body-force equivalent for seismic dislocations, Bull. Seismol. Soc. Amer. 54, 1854-1888 (1962)

[3] C. Callias and X. Markenscoff, Singular asymptotics of integrals and the near-field of a nonuniformly moving dislocation, Arch. Rational Mech. Anal. 102, 73-205 (1988)

[4] R. J. Clifton and X. Markenscoff, Elastic precursor decay and radiation from nonuniformly moving dislocations, J. Mech. Phys. Solids 29, 227-251 (1981)

[5] J. D. Eshelby, Uniformly moving dislocations, Proc. Phys. Soc. A62, 307-314 (1949)

[6] J. D. Eshelby, The Equation of motion of a dislocation, Phys. Rev. 90, 248-255 (1953)

[7] F. C. Frank, On the equation of motion of crystal dislocations, Proc. Phys. Soc. 62A, 131-134 (1949)

[8] H. Günther, Uberschallbewegung von Eigenspannungsguellen in der Kontinuumstheorie, Annalen der Physik 21, 93-105 (1968)

[9] J. Kuisalaas and T. Mura, On the motion of a screw dislocation, Recent Advances in Engng. Sci. (A. C. Eringen, ed.), Gordon and Breach, New York, 1964, pp. 543-563

[10] J. Kuisalaas and T. Mura, On the elastic field around an edge dislocation with application to dislocation vibration, Phil. Mag. 9, 1-7 (1964)

[11] R. Madariaga, Dynamics of an expanding circular fault, Bull. Seismol. Soc. Amer. 66, 639-666 (1976)

[12] X. Markenscoff, The transient motion of a nonuniformly moving dislocation, J. of Elasticity 10. 193-201 (1980) 
[13] X. Markenscoff and R. J. Clifton, The nonuniformly moving edge dislocation, J. Mech. Phys. Solids 29, 253-262 (1981)

[14] X. Markenscoff and R. J. Clifton, Radiation from expanding circular dislocation loops and elastic precursor decay, J. Appl. Mech. ASME 49, 792-796 (1982)

[15] X. Markenscoff and L. Ni, Nonuniform motion of an edge dislocation in an anisotropic solid, Quart. Appl. Math. 41, 475-494 (1984)

[16] X. Markenscoff, On the effect of dislocation loop curvature on elastic precursor decay, J. Appl. Mech. ASME 51, 753-758 (1984)

[17] X. Markenscoff and L. Ni, The singular nature of the stress field near an arbitrarily moving dislocation loop, J. Mech. Phys. Solids., (to appear in 1989)

[18] T. Mura, Continuous distributions of moving dislocations, Phil. Mag 8, 843-856 (1963)

[19] T. Mura, Methods of continuously distributed dislocations, Mathematical Theory of Dislocations (ASME) (T. Mura, ed.), 1969, pp. 25-48 